\title{
Main morbidities recorded in the women's international study of long duration oestrogen after menopause (WISDOM): a randomised controlled trial of hormone replacement therapy in postmenopausal women
}

Madge R Vickers, former head, MRC general practice research framework, ${ }^{1}$ Alastair H MacLennan, professor, department of obstetrics and gynaecology, ${ }^{2}$ Beverley Lawton, director women's health research centre, ${ }^{3}$ Deborah Ford, senior statistician, ${ }^{4}$ Jeannett Martin, former senior nurse manager, ${ }^{1}$ Sarah K Meredith, senior clinical epidemiologist, ${ }^{4}$ Bianca L DeStavola, reader in biostatistics, ${ }^{5}$ Sally Rose, research fellow, ${ }^{3}$ Anthony Dowell, professor, ${ }^{3}$ Helen C Wilkes, senior statistician, ${ }^{4}$ Janet H Darbyshire, director, ${ }^{4}$ Tom W Meade, emeritus professor, ${ }^{5}$ for the WISDOM team

${ }^{1}$ MRC General Practice Research Framework, Stephenson House, London NW1 2ND

'University of Adelaide, Women's and Children's Hospital, Adelaide SA 5006, Australia

${ }^{3}$ Department of Primary Health Care and General Practice, Wellington School of Medicine and Health Sciences,

New Zealand

${ }^{4}$ MRC Clinical Trials Unit, London NW1 2DA

${ }^{5}$ Department of Epidemiology and Population Health, London School of Hygiene and Tropical Medicine, London WC1E 7HT

Correspondence to:AHMacLennan alastair.maclennan@adelaide.edu.au

doi:10.1136/bmi.39266.425069.AD

\section{ABSTRACT}

Objective To assess the long term risks and benefits of hormone replacement therapy (combined hormone therapy versus placebo, and oestrogen alone versus combined hormone therapy).

Design Multicentre, randomised, placebo controlled, double blind trial.

Setting General practices in UK (384), Australia (91), and New Zealand (24).

Participants Postmenopausal women aged 50-69 years at randomisation. At early closure of the trial, 56583 had been screened, 8980 entered run-in, and 5692 (26\% of target of 22300) started treatment.

Interventions Oestrogen only therapy (conjugated equine oestrogens $0.625 \mathrm{mg}$ orally daily) or combined hormone therapy (conjugated equine oestrogens plus medroxyprogesterone acetate $2.5 / 5.0 \mathrm{mg}$ orally daily). Ten years of treatment planned.

Main outcome measures Primary outcomes: major cardiovascular disease, osteoporotic fractures, and breast cancer. Secondary outcomes: other cancers, death from all causes, venous thromboembolism, cerebrovascular disease, dementia, and quality of life. Results The trial was prematurely closed during recruitment, after a median follow-up of 11.9 months (interquartile range 7.1-19.6, total 6498 women years) in those enrolled, after the publication of early results from the women's health initiative study. The mean age of randomised women was 62.8 (SD 4.8) years. When combined hormone therapy $(n=2196)$ was compared with placebo $(n=2189)$, there was a significant increase in the number of major cardiovascular events $(7 \vee 0, \mathrm{P}=0.016)$ and venous thromboembolisms ( $22 v 3$, hazard ratio 7.36 (95\% Cl 2.20 to 24.60$)$ ). There were no statistically significant differences in numbers of breast or other cancers ( $22 v 25$, hazard ratio 0.88 (0.49 to 1.56$)$ ), cerebrovascular events $(14 \vee 19,0.73$ (0.37 to 1.46$))$, fractures $(40 v 58,0.69$ ( 0.46 to 1.03$))$, and overall deaths
( $8 v 5,1.60$ (0.52 to 4.89)). Comparison of combined hormone therapy $(n=815)$ versus oestrogen therapy $(n=826)$ outcomes revealed no significant differences. Conclusions Hormone replacement therapy increases cardiovascular and thromboembolic risk when started many years after the menopause. The results are consistent with the findings of the women's health initiative study and secondary prevention studies. Research is needed to assess the long term risks and benefits of starting hormone replacement therapy near the menopause, when the effect may be different. Trial registration Current Controlled Trials ISRCTN 63718836

\section{INTRODUCTION}

Although the use of hormone replacement therapy for control of moderate to severe menopausal symptoms is well established, its long term use for disease prevention in postmenopausal women is in dispute. ${ }^{1-3}$ Ten randomised controlled trials have investigated the risks and benefits of hormone replacement therapy in postmenopausal women. ${ }^{4-13}$ Three trials in the United States, ${ }^{4712}$ two in the United Kingdom, ${ }^{89}$ and one in Estonia $^{13}$ showed that such therapy does not protect against development of cardiovascular disease and may increase the risk.

In the largest trial, and the only one designed to assess the prevention of cardiovascular disease, the US women's health initiative study, women aged 50-79 years taking combined oestrogen and progestogen had a significantly increased risk of stroke, pulmonary embolism, and breast cancer and a decreased risk of hip fracture and colorectal cancer compared with women taking placebo. ${ }^{7}$ This study found that combined oestrogen and progestogen therapy might increase coronary events in older women (aged 7079) in their first year of treatment. ${ }^{14}$ Overall, the risks seen in the women's health initiative study were likely 


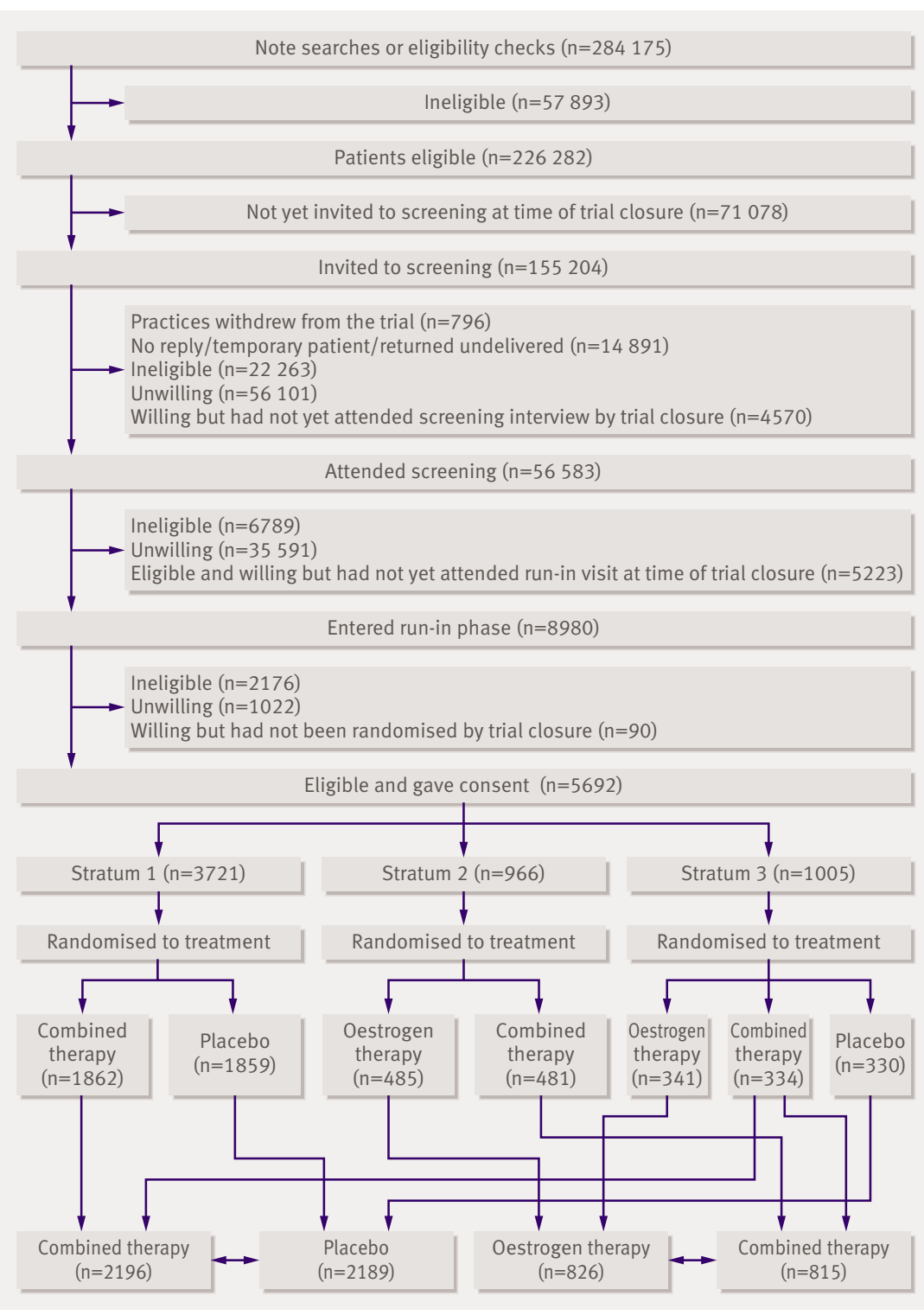

Flow of patients through the women's international study of long duration oestrogen after menopause (WISDOM). (Trial closure was on 22 October 2002. Combined therapy=conjugated equine oestrogen $0.625 \mathrm{mg}+$ medroxyprogesterone acetate $2.5 \mathrm{mg}$ orally daily. Oestrogen therapy=conjugated equine oestrogen $0.625 \mathrm{mg}$ orally daily)

to outweigh the benefits, and the combined oestrogen and progestogen arm of the trial was closed prematurely after a mean of 5.2 years of follow-up. Later, the oestrogen only arm of the trial in women who had had a hysterectomy was also closed prematurely, after an average of 6.8 years of follow-up, as it showed an increased risk of stroke but no overall difference in cardiovascular disease or breast cancer. ${ }^{12}$

In 1989 the UK Medical Research Council agreed that a randomised controlled trial to assess the long term benefits and risks of hormone replacement therapy was a priority. Successful feasibility trials were conducted, and, after extensive review, funding from multiple sources was secured for the women's international study of long duration oestrogen after menopause (WISDOM), which began recruitment in
$1999 .{ }^{1516}$ The women's health initiative study had begun in 1997, enrolling women aged 50-79 for an expected average of 8.5 years of follow-up. WISDOM was originally designed to investigate a younger age group (45-60 years old) to ensure the data were relevant to the normal use of hormone replacement therapy, but this was later modified to 50-64 years and then extended to 69 years to increase the likelihood of relevant events during the trial planned for 10 years treatment with a further 10 years' follow-up (later modified to five years).$^{16}$ The aim was to recruit 22300 postmenopausal women and to assess the balance of long term risks and benefits of hormone replacement therapy with particular emphasis on cardiovascular disease and dementia. The design allowed for two main comparisons: combined oestrogen and progestogen therapy versus placebo and, in women who had had a hysterectomy, oestrogen alone versus combined oestrogen and progestogen therapy.

Recruitment began in the UK in 1999 and in Australia and New Zealand in 2000. Recruitment was still under way when the MRC stopped the trial after the first results of the combined oestrogen and progestogen arm of the women's health initiative study were published in 2002. This paper presents the main clinical outcomes for WISDOM after 6498 person years of follow-up for a median of 11.9 months.

\section{METHODS}

\section{Setting}

Recruitment took place in general practice. In the UK participants were recruited through 384 general practices from the MRC General Practice Research Framework. In addition, 91 general practices in Australia and 24 in New Zealand participated.

\section{Participants}

Full details of recruitment procedures and other methods are published elsewhere. ${ }^{17}$ In each country we identified women aged 50-69 years from practice registers. Where possible, patients' notes were searched by a research nurse to exclude ineligible women. Postmenopausal women (no menstrual period in the past 12 months or had undergone hysterectomy) were eligible for the trial.

The exclusion criteria are described in full elsewhere. ${ }^{17}$ Main exclusion criteria were a history of breast cancer; any other cancer in the past 10 years except basal and squamous cell skin cancer; endometriosis or endometrial hyperplasia; venous thromboembolism; gall bladder disease in women who had not had a cholecystectomy; and myocardial infarction, unstable angina, cerebrovascular accident, subarachnoid haemorrhage, transient ischaemic attack, or use of hormone replacement therapy within the past six months. Women taking hormone replacement therapy at screening who were prepared to enter the placebo controlled strata of the study agreed to stop the therapy for three months before the run-in phase. During runin they took placebo so that at randomisation they had not taken hormone replacement therapy for six 
months. Women who were, in the opinion of their general practitioner, unlikely to be able to give informed consent or successfully complete trial procedures were also excluded.

Eligible women and those whose notes were not available were contacted by post and invited to attend a screening session with a research nurse at their practice. In Australia patients were invited first to group sessions to learn more about hormone replacement therapy and the trial before volunteering for screening, whereas in the UK and New Zealand the research nurse discussed these details at the interview. The strategy was to recruit the oldest women first. Women completed a screening questionnaire, were provided with written information, and, if, after consideration for two weeks, they were willing to participate, provided written consent, and entered a 12 week run-in period (figure). All women took medication during run-in: women who were willing to enter a placebo controlled randomisation took placebo and women who had undergone a total hysterectomy and were willing only to enter a comparison of two active treatments took oestrogen only. Four weeks before the end of run-in, eligible women who had achieved $80 \%$ compliance were invited to enter the trial and were randomised to treatment (figure).

\section{Method of random allocation}

Treatment was randomly allocated centrally with a computer based, stratified block randomisation program. Stratification was based on hysterectomy status and intended use of hormone replacement therapy. Women with a uterus or subtotal hysterectomy (stratum 1 of the study) were randomised to combined oestrogen plus progestogen or to placebo using a block size of 16 . Women with no uterus and unwilling to take placebo were randomised to either oestrogen only or combined oestrogen and progestogen therapy (stratum 2) using a block size of 16, and those willing to enter a placebo controlled comparison were randomised to oestrogen, combined oestrogen plus progestogen, or placebo using a block size of 24 (stratum 3). Within each stratum there was equal probability of allocation to any of the treatment arms.

As soon as the woman was randomised the appropriate treatment pack was issued from the central store, labelled for the participant and dispatched to the practice. Drugs were packed in foils containing tablets for 28 days. Within each stratum the drug packaging for each arm of the trial was identical. Randomised treatment was started if, at the end of run-in, the woman remained eligible and willing to enter the trial.

\section{Interventions}

The oestrogen therapy was conjugated equine oestrogens (Premarin, Wyeth Ayerst US), $0.625 \mathrm{mg}$ orally daily. The combined therapy was conjugated equine oestrogens as above plus medroxyprogesterone acetate $2.5 \mathrm{mg}$ orally daily (Prempro, Wyeth Ayerst US). Women with a uterus and within three years of their last period, those aged 50-53, and older women with unacceptable breakthrough bleeding took $5.0 \mathrm{mg}$ medroxyprogesterone acetate (Premique, Wyeth Ayerst US). Women with a uterus who experienced unacceptable spotting or bleeding with the combined therapy containing $5.0 \mathrm{mg}$ medroxyprogesterone acetate were offered open label Premique cycle (Premarin $0.625 \mathrm{mg}$ orally daily plus medroxyprogesterone acetate $10 \mathrm{mg}$ orally for the last 14 days of a 28 day cycle).

The planned median treatment duration of the trial was 10 years (range 9-12), with treatment being coterminous in all participants. As far as possible, the trial was conducted in a double-blind manner, though full blindness could not be maintained when vaginal bleeding triggered a code break and investigation for possible pathology.

\section{Data collection}

Women were to be seen at 4, 14, 27, 40, and 52 weeks after start of treatment and then at six month intervals. A final visit took place as soon as possible after the closure of the trial. At the start of treatment and at each follow-up visit, information was collected on all outcomes, adverse events, and other medical history to check that patients remained eligible. The research nurse entered data directly on to a customised Access database that incorporated extensive checks for plausibility, consistency, and completeness. Data were sent by modem link (UK) or via a standard File Transfer Program server site (Australia and New Zealand) to the coordinating centre within one week of collection. A member of the study team, who was blinded to treatment allocation, obtained any data needed to confirm a clinical event from the general practice, hospital, or coroner.

\section{Main clinical outcome measures}

Primary outcomes were major cardiovascular disease (defined as one or more of unstable angina requiring hospitalisation, fatal or non-fatal myocardial infarction, or sudden coronary death), osteoporotic fractures (all fractures other than of the skull, face, cervical spine, fingers, or toes), and breast cancer. Secondary outcomes were breast cancer mortality, other cancers, death from all causes, venous thromboembolism (deep vein thrombosis, pulmonary embolism, or retinal vein occlusion), cerebrovascular disease, and dementia. Quality of life and psychological wellbeing were measured and are reported separately. All outcomes were reviewed blind to treatment allocation. All cardiovascular and cancer outcomes and 10\% of fractures were reviewed by independent assessors.

Participants were asked about symptoms and adverse events at each visit. Each adverse event was assessed for seriousness according to defined criteria in the protocol. Reporting of certain potential complications of hormone replacement therapysuch as gallbladder disease, sudden loss of vision, or diplopia-required the nurse to interrupt treatment temporarily. 


\section{Sample size}

WISDOM was designed to detect a $25 \%$ reduction in the number of cases of coronary heart disease (excluding unstable angina) and stroke over 10 years comparing combined oestrogen and progestogen therapy with placebo in women aged 50-64 years at randomisation. A total sample size of 18000 , assuming 13000 were taking combined therapy or placebo in strata 1 and 3, provided $80 \%$ power at the $5 \%$ significance level.

The primary outcome was subsequently changed to exclude stroke and to include unstable angina, and the age range at randomisation was extended to 69 years.
Other assumptions were modified. In particular, cardiovascular event rates were reduced as these were falling faster than expected in the populations of the countries involved and estimates of withdrawal rates were revised. The expected maximum recruitment of 22300 (16000 for combined therapy versus placebo) provided $80 \%$ power at the $5 \%$ significance level to detect a $29 \%$ reduction from an expected probability of a primary outcome event in the placebo group during the trial period of 39 per 1000 women randomised. This sample size also had the power to detect a $20 \%$ reduction, from an expected probability of 95 per

\begin{tabular}{|c|c|c|c|c|}
\hline \multirow[b]{2}{*}{ Participant characteristics } & \multicolumn{2}{|c|}{ Combined therapy $v$ placebo* } & \multicolumn{2}{|c|}{$\begin{array}{c}\text { Combined therapy } v \text { oestrogen } \\
\text { therapy } \dagger\end{array}$} \\
\hline & $\begin{array}{l}\text { Combined therapy } \\
\qquad(n=2196)\end{array}$ & Placebo $(n=2189)$ & $\begin{array}{l}\text { Combined } \\
\text { therapy }(n=815)\end{array}$ & $\begin{array}{l}\text { Oestrogen therapy } \\
\qquad(\mathrm{n}=826)\end{array}$ \\
\hline \multicolumn{5}{|l|}{ Sociodemographic variables } \\
\hline Mean (SD) age at randomisation (years) & $63.3(4.7)$ & $63.3(4.6)$ & $61.7(5.1)$ & $61.9(5.1)$ \\
\hline \multicolumn{5}{|l|}{ Age at randomisation: } \\
\hline $50-54$ years & $145(7)$ & $131(6)$ & $93(11)$ & $96(12)$ \\
\hline $55-59$ years & 395 (18) & 419 (19) & $219(27)$ & $205(25)$ \\
\hline $60-64$ years & $716(33)$ & $732(33)$ & $262(32)$ & $268(32)$ \\
\hline$\geq 65$ years & $938(43)$ & $906(41)$ & $241(30)$ & $257(31)$ \\
\hline Mean (SD) years since menopause (last menstruation) & $14.8(7.2)$ & $14.7(7.1)$ & $18.2(7.4)$ & $17.9(7.5)$ \\
\hline Non-white ethnic status & $23 / 2174$ (1) & $30 / 2163(1)$ & $16 / 802(2)$ & $16 / 815(2)$ \\
\hline Left full time education before 16 years old & $1607(73)$ & $1564(71)$ & $611(75)$ & $615(75)$ \\
\hline Using HRT at screening: & $167(8)$ & $184(8)$ & $430(53)$ & $429(52)$ \\
\hline Median (interquartile range) duration of use (years) & $8(5.3-11)$ & $10(4.6-10.2)$ & $10(6.6-12)$ & $10(6.5-12.7)$ \\
\hline Ever used HRT at screening: & $1041(47)$ & $1005(46)$ & $646(79)$ & 648 (79) \\
\hline Median (interquartile range) duration of use (years) & $3.8(0.8-8)$ & $4(0.9-8)$ & $8(4-11.4)$ & $8(4.1-1.3)$ \\
\hline \multicolumn{5}{|l|}{ Cardiovascular risk factors } \\
\hline Mean (SD) body mass index & $27.9(4.9)$ & $28.0(5.2)$ & $28.0(4.7)$ & $27.9(5.0)$ \\
\hline \multicolumn{5}{|l|}{ Body mass index: } \\
\hline$<25$ & $629(29)$ & $659(30)$ & $231(28)$ & $250(30)$ \\
\hline $25-29$ & $934(43)$ & $848(39)$ & $356(44)$ & $330(40)$ \\
\hline$\geq 30$ & $623(28)$ & $675(31)$ & $226(28)$ & $243(30)$ \\
\hline Mean (SD) systolic blood pressure (mm Hg) & $136(21)$ & $137(22)$ & $137(21)$ & $135(20)$ \\
\hline Mean (SD) diastolic blood pressure (mm Hg) & $73(10)$ & $73(10)$ & $74(10)$ & $73(10)$ \\
\hline Current smoker & $256(12)$ & 309 (14) & $89(11)$ & $118(14)$ \\
\hline Former smokerł: & $664(30)$ & $633(29)$ & $256(31)$ & $238(29)$ \\
\hline Median (interquartile range) time since stopping (years) & $18(9-29)$ & $18(9-28)$ & $18(8-26)$ & $20(9-30)$ \\
\hline Previous angina & $127(6)$ & $104(5)$ & $51(6)$ & $41(5)$ \\
\hline Previous myocardial infarction & $40(2)$ & $26(1)$ & $17(2)$ & $10(1)$ \\
\hline Previous stroke & $30(1)$ & $38(2)$ & $16(2)$ & $12(1)$ \\
\hline History of diabetes & $70(3)$ & $93(4)$ & $28(3)$ & $30(4)$ \\
\hline \multicolumn{5}{|l|}{ Breast cancer risk factors } \\
\hline 1st degree female relative with breast cancer & $149 / 1954(8)$ & $183 / 1956(9)$ & $67 / 723(9)$ & $59 / 740(8)$ \\
\hline Current non-drinker & $354(16)$ & $332(15)$ & $120(15)$ & $132(16)$ \\
\hline \multicolumn{5}{|l|}{ Osteoporotic fracture risk factors } \\
\hline Previous fracture past age of 50 & $390(18)$ & $397(18)$ & $110(13)$ & $118(14)$ \\
\hline Maternal osteoporosis & $179 / 2161(8)$ & $166 / 2138(8)$ & $77 / 793(10)$ & $87 / 812(11)$ \\
\hline \multicolumn{5}{|l|}{ Overall quality of life } \\
\hline Mean (SD) visual anologue scale (of 0-100) & $78.5(14)$ & $77.7(14)$ & 77.6 (15) & 77.0 (15) \\
\hline
\end{tabular}

All variables are affected by missing values. HRT = hormone replacement therapy.

${ }^{*}$ Combined therapy $=$ conjugated equine oestrogen $0.625 \mathrm{mg}+$ medroxyprogesterone acetate $2.5 \mathrm{mg}$ orally daily.

tOestrogen therapy $=$ conjugated equine oestrogen $0.625 \mathrm{mg}$ orally daily.

‡Number of past smokers who had recorded a time since stopping smoking. 
1000 women, in all osteoporotic fractures and a $40 \%$ increase, from an expected probability of 36 per 1000 women, in breast cancer.

The predicted withdrawal from randomised treatment was 36\% (10\% in the first year of treatment, $7 \%$ in year $2,4 \%$ in year $3,3 \%$ in year 4 , and $2 \%$ in years $5-10$ ), and the associated attenuation of observed differences between randomised groups was taken into account in the power calculations. Based on feasibility studies and current use of hormone replacement therapy, it was assumed that $20 \%$ of women would be $65-69$ years with $80 \%$ distributed equally across the three five-year age bands 50-54, 55-59, and 60-64 years. Full details of the assumptions made for the event rates in the placebo group are reported elsewhere. ${ }^{17}$

\section{Statistical methods}

Follow-up time for each participant was calculated for each outcome separately from date of randomisation until the date of outcome, of death, of loss to follow-up, or of trial closure, whichever occurred first. The outcomes of interest were cardiovascular disease, cerebrovascular disease, venous thromboembolism, fractures, cancer, and death. We adopted the intention-to-treat principle when assessing treatment effects, with $\mathrm{P}<0.05$ used to define statistical significance. We classified participants according to randomisation group and compared combined oestrogen plus progestogen therapy with placebo (pooling data from women from strata 1 and 3), and combined therapy with oestrogen only therapy (combining strata 2 and 3 ). Women from stratum 3 who were randomised to combined therapy were thereby included in both comparisons.

To account for the prospective nature of the data, we calculated event rates (per 10000 women-years) as the number of events divided by the relevant accumulated person-time - that is, assuming a Poisson regression (constant hazards) model. We calculated hazard ratios under the more flexible Cox proportional hazards model, after graphically checking its assumptions. ${ }^{18}$ The results are reported as, respectively, rates and hazard ratios for the effect of combined therapy versus either placebo or oestrogen therapy (with 95\% confidence intervals), with associated likelihood ratio tests for significance. ${ }^{18}$ No adjustment was made for multiple a priori testing.

\begin{tabular}{|c|c|c|}
\hline Characteristics & Randomised $(n=5692)$ & Screened $(n=56468)$ \\
\hline \multicolumn{3}{|l|}{ Sociodemographic variables } \\
\hline Mean (SD) age at randomisation (years) & $62.9(4.8)$ & $61.4(4.9)$ \\
\hline \multicolumn{3}{|l|}{ Age at randomisation: } \\
\hline $50-54$ years & $437(8)$ & $8390(15)$ \\
\hline $55-59$ years & $1177(21)$ & $14768(26)$ \\
\hline 60-64 years & $1876(33)$ & $19087(34)$ \\
\hline$\geq 65$ years & $2199(39)$ & $14223(25)$ \\
\hline Non-white ethnic status & $80 / 5623(1)$ & $1174 / 54008(2)$ \\
\hline Using HRT at screening: & $1175(21)$ & $17578(31)$ \\
\hline Median (interquartile range) duration of use (years) & $9(6-12)$ & $9(5.2-12)$ \\
\hline Ever used HRT at screening: & $3144(55)$ & $32309(57)$ \\
\hline Median (interquartile range) duration of use (years) & $5.3(1.5-10)$ & $6.2(2-10)$ \\
\hline \multicolumn{3}{|l|}{ Cardiovascular risk factors } \\
\hline Mean (SD) body mass index & $28.0(5.0)$ & $27.4(5.0)$ \\
\hline Mean (SD) systolic blood pressure (mm Hg) & $136.2(21)$ & $142(23)$ \\
\hline Mean (SD) diastolic blood pressure $(\mathrm{mm} \mathrm{Hg})$ & $73.1(10)$ & $76(11)$ \\
\hline Current smoker & $738(13)$ & $6794(12)$ \\
\hline Former smoker & $1782(31)$ & $15095(27)$ \\
\hline Previous unstable angina & $297(5)$ & $2919(5)$ \\
\hline Previous myocardial infarction & $84(1)$ & $887(2)$ \\
\hline Previous stroke & $90(2)$ & $1261(2)$ \\
\hline History of diabetes & $210(4)$ & $1932(3)$ \\
\hline \multicolumn{3}{|l|}{ Breast cancer risk factors } \\
\hline 1 st degree female relative with breast cancer & $434 / 5080(9)$ & $4755 / 50372(9)$ \\
\hline Current non-drinker & $882(16)$ & $10321(18)$ \\
\hline \multicolumn{3}{|l|}{ Osteoporotic fracture risk factors } \\
\hline Previous fracture past age of 50 & $959(17)$ & 7405 (13) \\
\hline \multicolumn{3}{|l|}{ Overall quality of life } \\
\hline Mean (SD) visual anologue scale (of 0-100) & $77.9(14)$ & $76.0(16)$ \\
\hline
\end{tabular}




\section{RESULTS}

\section{Recruitment}

A total of 284175 women aged 50-69 years were registered at the participating practices $(224075$ from the UK, 36210 from Australia, and 23890 from New Zealand), of whom 226282 were potentially eligible for the trial (figure). When the trial closed on 22 October 2002,155204 women $(68 \%$ of those potentially eligible) had been invited to screening, 56583 had attended, and 4570 had agreed to attend but had not yet been interviewed. Of those screened and eligible, 14203 $(29 \%)$ agreed to enter the run-in phase, of whom 8980 had entered at the time of closure. At the end of run-in, 5692 participants remained eligible, gave consent, were randomised, and started trial treatment $(26 \%$ of the target of $22300 ; 5250$ from the UK, 319 from Australia, and 123 from New Zealand).

\section{Baseline data}

The participants' mean age was 62.8 years (SD 4.8), reflecting the strategy of recruiting older women first. Women who had undergone a hysterectomy were, on average, slightly younger and were more likely to have ever used hormone replacement therapy and to have used it for a longer time than those women with a uterus. Women who were not willing to accept placebo randomisation (stratum 2) were more likely to have used hormone replacement therapy and to be using it at screening $(82 \%)$. Other characteristics were similar for the three strata. The characteristics of the treatment groups for both the comparison groups of combined therapy versus placebo and oestrogen therapy versus combined therapy were also similar (table 1).

In the main treatment comparison (combined therapy versus placebo) $8 \%$ in each group were using hormone replacement therapy at screening and $46-47 \%$ of those screened were past users of hormone replacement therapy for a median of 3.8-4 years. Data from the screening interviews indicate that, with regard to major risk factors for the primary outcomes, WISDOM participants were similar to women screened in the participating practices (table 2), although screened women had greater current use of hormone replacement therapy $(31 \%)$ than randomised women $(21 \%)$. The mean number of years since menopause (last menstrual period) in all women with a uterus was 13.8 (SD 6.7). The mean number of years since last menstrual period or hysterectomy with or without oophorectomy in all women without a uterus (strata 2 and 3) was 18.5 (SD 7.5). In the main comparison of combined therapy versus placebo, which included women with and without a uterus from strata 1 and 3 , the mean years from last menstrual period were 14.8 and 14.7 respectively.

\section{Follow up}

With the early closure of the trial the median follow-up time was 11.9 months (interquartile range 7.3-19.6), with a total follow-up time of 6498 women years. In women randomised to combined therapy or placebo, median follow-up was 12.8 (7.5-20.4) months, with a total of 5214 person-years; for women randomised to combined therapy or oestrogen therapy the figures were 10.3 (6.4-16.8) months and 1688 years, respectively. Five participants were lost to follow-up.

By 15 July 2002, when the results from the women's health initiative study were disseminated, 725 women $(12.7 \%)$ had withdrawn from randomised treatment; most were in stratum 1 (369 taking combined therapy; 162 taking placebo) or stratum 3 (46 taking combined therapy; 50 taking oestrogen therapy, and 38 taking placebo). Table 3 gives the reasons for withdrawal. An additional 368 women were taking a temporary interruption of trial treatment at the time the women's health initiative reported; of these, only 23 restarted randomised treatment before trial closure.

\begin{tabular}{|c|c|c|c|c|}
\hline \multirow[b]{2}{*}{ Reasonsł } & \multicolumn{2}{|c|}{ Combined therapy $v$ placebo* } & \multicolumn{2}{|c|}{ Combined therapy $v$ oestrogen therapy $\dagger$} \\
\hline & $\begin{array}{l}\text { Combined therapy } \\
\qquad(n=430)\end{array}$ & Placebo $(n=203)$ & $\begin{array}{l}\text { Combined therapy } \\
\qquad(n=88)\end{array}$ & $\begin{array}{l}\text { Oestrogen therapy } \\
\qquad(n=78)\end{array}$ \\
\hline Vaginal bleeding & $122(28)$ & $2(1)$ & 0 & 0 \\
\hline Other potentially HRT related symptoms & $94(22)$ & $18(9)$ & $23(27)$ & $14(18)$ \\
\hline Concerns about risks of HRT & $33(8)$ & $17(8)$ & $8(9)$ & $10(13)$ \\
\hline Social (such as moved) & $22(5)$ & $24(12)$ & $6(7)$ & $10(13)$ \\
\hline No longer wished to be in a trial & $16(4)$ & $33(16)$ & $5(6)$ & $1(1)$ \\
\hline Protocol excluded outcome & $22(5)$ & $5(2)$ & $6(7)$ & $2(3)$ \\
\hline Other protocol exclusion & $3(1)$ & $3(1)$ & 0 & $1(1)$ \\
\hline Cardiac outcome & $5(1)$ & $1(0.5)$ & $1(1)$ & $1(1)$ \\
\hline Cerebrovascular outcome & $2(0.5)$ & $6(3)$ & $1(1)$ & $1(1)$ \\
\hline Menopausal symptoms & $2(0.5)$ & $10(5)$ & $1(1)$ & $1(1)$ \\
\hline Non-trial HRT prescription & $1(0)$ & $3(1)$ & $3(3)$ & $3(4)$ \\
\hline Other diagnoses or symptoms & $103(24)$ & $66(33)$ & $28(33)$ & $30(38)$ \\
\hline Unknown & $5(1)$ & $15(7)$ & $4(5)$ & $2(3)$ \\
\hline
\end{tabular}

HRT = hormone replacement therapy.

${ }^{*}$ Combined therapy $=$ conjugated equine oestrogen $0.625 \mathrm{mg}+$ medroxyprogesterone acetate $2.5 \mathrm{mg}$ orally daily.

tOestrogen therapy $=$ conjugated equine oestrogen $0.625 \mathrm{mg}$ orally daily.

$\ddagger$ More than one reason may be given. 
Table 4 | Primary and secondary clinical outcomes in WISDOM by time of follow-up and randomised treatment*

\begin{tabular}{|c|c|c|c|c|c|}
\hline \multirow[b]{2}{*}{ Outcomes } & \multirow[b]{2}{*}{ All $(n=5692)$} & \multicolumn{2}{|c|}{ Combined therapy $v$ placebo } & \multicolumn{2}{|c|}{ Combined therapy $v$ oestrogen therapy } \\
\hline & & $\begin{array}{l}\text { Combined therapy } \\
\quad(n=2196)\end{array}$ & Placebo $(n=2189)$ & $\begin{array}{l}\text { Combined therapy } \\
\qquad(\mathrm{n}=815)\end{array}$ & $\begin{array}{l}\text { Oestrogen therapy } \\
\quad(n=826)\end{array}$ \\
\hline \multicolumn{6}{|l|}{ Cardiovascular disease } \\
\hline Unstable angina & 6 & 3 & 0 & 3 & 1 \\
\hline Non-fatal myocardial infarct & 6 & 4 & 0 & 1 & 2 \\
\hline Fatal myocardial infarct & 0 & 0 & 0 & 0 & 0 \\
\hline Sudden coronary death & 1 & 0 & 0 & 0 & 1 \\
\hline Any of the above & 11 & 7 & 0 & 4 & 2 \\
\hline Rate $(95 \% \mathrm{Cl}) \dagger$ & $17.0(9.4$ to 30.6$)$ & $26.9(12.8$ to 56.4$)$ & 0 & $47.8(18.0$ to 127.5$)$ & $23.6(5.9$ to 94.4$)$ \\
\hline Hazard ratio $(95 \% \mathrm{Cl})$; P value & & \multicolumn{2}{|c|}{ NA; 0.016} & \multicolumn{2}{|c|}{2.03 (0.37 to 11.09$) ; 0.40$} \\
\hline \multicolumn{6}{|l|}{ Cerebrovascular disease } \\
\hline Non-fatal & 36 & 13 & 19 & 4 & 4 \\
\hline Fatal & 1 & 1 & 0 & 0 & 0 \\
\hline Fatal or non-fatal & 37 & 14 & 19 & 4 & 4 \\
\hline Rate $(95 \% \mathrm{Cl}) \dagger$ & $57.2(41.4$ to 78.9$)$ & 53.8 (31.9 to 90.9$)$ & $73.4(46.8$ to 115.0$)$ & $47.9(18.0$ to 127.6$)$ & 47.1 (17.7 to 125.6$)$ \\
\hline Hazard ratio $(95 \% \mathrm{Cl})$; P value & & \multicolumn{2}{|c|}{0.73 (0.37 to 1.46$) ; 0.38$} & \multicolumn{2}{|c|}{1.01 (0.25 to 4.04$) ; 0.99$} \\
\hline \multicolumn{6}{|l|}{ Venous thromboembolism } \\
\hline Deep vein thrombosis & 18 & 13 & 1 & 5 & 3 \\
\hline Pulmonary embolism & 14 & 10 & 2 & 2 & 1 \\
\hline Fatal thromboembolism & 3 & 2 & 0 & 0 & 1 \\
\hline Any of the above & 30 & 22 & 3 & 7 & 3 \\
\hline Rate $(95 \% \mathrm{Cl}) \dagger$ & 46.4 (32.4 to 66.3$)$ & 85.1 (56.0 to 129.2 ) & 11.5 (3.7 to 35.7$)$ & $84.3(40.2$ to 176.9$)$ & 35.3 (11.4 to 109.5$)$ \\
\hline Hazard ratio $(95 \% \mathrm{Cl})$; P value & & \multicolumn{2}{|c|}{$7.36(2.20$ to 24.60$) ;<0.001$} & \multicolumn{2}{|c|}{2.39 (0.62 to 9.24$) ; 0.19$} \\
\hline \multicolumn{6}{|l|}{ Osteoporotic fractures } \\
\hline Hip & 5 & 2 & 3 & 1 & 0 \\
\hline Other & 108 & 38 & 55 & 11 & 8 \\
\hline Any & 113 & 40 & 58 & 12 & 8 \\
\hline Rate $(95 \% \mathrm{Cl}) \dagger$ & $\begin{array}{c}176.1(146.5 \text { to } \\
211.8)\end{array}$ & $\begin{array}{l}155.3(114.0 \text { to } \\
211.8)\end{array}$ & $\begin{array}{l}226.2(174.9 \text { to } \\
292.6)\end{array}$ & 144.5 (82.1 to 254.4$)$ & 94.4 (47.2 to 188.8$)$ \\
\hline Hazard ratio $(95 \% \mathrm{Cl})$; P value & & \multicolumn{2}{|c|}{0.69 (0.46 to 1.03$) ; 0.07$} & \multicolumn{2}{|c|}{1.52 (0.62 to 3.72$) ; 0.35$} \\
\hline \multicolumn{6}{|l|}{ Cancer } \\
\hline Breast & 16 & 5 & 7 & 3 & 2 \\
\hline Colorectal & 6 & 2 & 2 & 1 & 2 \\
\hline Other & 37 & 15 & 16 & 4 & 3 \\
\hline Any & 59 & 22 & 25 & 8 & 7 \\
\hline Rate $(95 \% \mathrm{Cl}) \dagger$ & 91.3 (70.7 to 117.8$)$ & $84.8(55.8$ to 128.7$)$ & 96.5 (65.2 to 142.8$)$ & 96.0 (48.0 to 192.0$)$ & 82.8 (39.5 to 173.6$)$ \\
\hline Hazard ratio $(95 \% \mathrm{Cl})$; P value & & \multicolumn{2}{|c|}{0.88 (0.49 to 1.56$) ; 0.65$} & \multicolumn{2}{|c|}{1.16 (0.42 to 3.20$) ; 0.78$} \\
\hline \multicolumn{6}{|l|}{ Death } \\
\hline Breast cancer & 0 & 0 & 0 & 0 & 0 \\
\hline Colorectal cancer & 1 & 1 & 0 & 1 & 0 \\
\hline Other cancer & 5 & 2 & 3 & 0 & 0 \\
\hline Cardiovascular & 1 & 0 & 0 & 0 & 1 \\
\hline Cerebrovascular & 1 & 1 & 0 & 0 & 0 \\
\hline Venous thromboembolism & 3 & 2 & 0 & 0 & 1 \\
\hline Other causes & 4 & 2 & 2 & 0 & 0 \\
\hline All death & 15 & 8 & 5 & 1 & 2 \\
\hline Rate $(95 \% \mathrm{Cl}) \dagger$ & 23.1 (13.9 to 38.3$)$ & $30.7(15.3$ to 61.3$)$ & $19.2(8.0$ to 46.1$)$ & $11.9(1.7$ to 84.7$)$ & $23.5(5.9$ to 94.0$)$ \\
\hline Hazard ratio $(95 \% \mathrm{Cl})$; P value & & \multicolumn{2}{|c|}{1.60 (0.52 to 4.89$) ; 0.40$} & $0.51(0.05 t$ & 5.58); 0.57 \\
\hline Any event & 242 & 99 & 104 & 35 & 23 \\
\hline Rate $(95 \%$ Cl)† & $\begin{array}{c}382.7(337.4 \text { to } \\
434.0)\end{array}$ & $\begin{array}{c}390.9(321.0 \text { to } \\
476.1)\end{array}$ & $\begin{array}{c}410.9(339.0 \text { to } \\
497.9)\end{array}$ & $\begin{array}{c}429.9(308.6 \text { to } \\
598.7)\end{array}$ & $274.6(182.5$ to 413.2$)$ \\
\hline Hazard ratio $(95 \%$ Cl); P value & & $0.95(0.72 t$ & 1.25); 0.72 & $1.56(0.92 t$ & o 2.64); 0.09 \\
\hline
\end{tabular}


During follow-up of women randomised to combined therapy or placebo, trial treatment was supplied for $73 \%$ of time at risk in women allocated to active treatment $(27 \%$ of time at risk was after withdrawal or during a temporary interruption of treatment) and for $86 \%$ of time at risk in women allocated to placebo. Corresponding proportions in women randomised between combined therapy and oestrogen therapy were $83 \%$ and $84 \%$ respectively.

The treatment code was unblinded in only two of the 1971 women who had undergone hysterectomy, but in women with a uterus the proportion unblinded was high, mostly as a result of vaginal bleeding in those randomised to combined therapy, where 712/ $1862(38 \%)$ were unblinded, compared with 66/1859 $(4 \%)$ of those randomised to placebo (hazard ratio $13.4(95 \%$ confidence interval 10.4 to 17.3$)$, $\mathrm{P}<0.001)$.

\section{Clinical outcomes}

The total number of events for all trial outcomes was low because the trial was stopped early. There are no data on dementia because the first follow-up assessment was planned for two years after randomisation.

\section{Combined oestrogen and progestogen therapy versus placebo}

Compared with those taking placebo, women taking combined therapy had significantly increased rates of cardiovascular events (26.9 $v 0$ per 10000 womenyears, $\mathrm{P}=0.016)$ and venous thromboembolism $(85.1$ $v 11.5$ per 10000 women-years, hazard ratio 7.36 (2.20 to 24.60$), \mathrm{P}<0.001)$ and a non-significant reduction in the rate of osteoporotic fractures (155.3 v 226.2 per 10000 women-years, hazard ratio 0.69 (0.46 to 1.03), $\mathrm{P}=0.07$ ) (table 4). Rates for cerebrovascular disease, breast cancer, and other cancers were not significantly different in the two groups.

\section{Cardiovascular events}

The 11 cardiovascular events recorded were all in women randomised to hormone replacement therapy (nine to combined therapy and two to oestrogen therapy). All but two of these women were over 64 years of age at trial entry and had one or more cardiovascular risk factors (three had a history of myocardial infarction or angina, two had diabetes, four smoked, and eight had a body mass index $\geq 25$ ).

\section{Cerebrovascular events}

There was no significant difference in cerebrovascular events with a rate of 73.4 (95\% confidence interval 46.8 to 115.0$)$ per 10000 women-years in the placebo group and 53.8 (31.9 to 90.9$)$ in the combined therapy group (hazard ratio 0.73 ( 0.37 to 1.46$), \mathrm{P}=0.38$ ). When transient ischaemic attacks were excluded the differences between the treatment groups were even smaller, with a hazard ratio of 0.91 ( 0.39 to 2.14 ) for combined therapy versus placebo and oestrogen therapy and 1.01 (0.21 to 5.02) for combined therapy versus oestrogen therapy.

\section{Oestrogen and progestogen versus oestrogen alone}

The numbers of participants and the number of events in this comparison are much smaller than for the comparison of combined oestrogen plus progestogen versus placebo (table 4). There is a suggestion in the combined therapy group of an increase in cardiovascular events (47.8 $v 23.6$ per 10000 women-years, hazard ratio 2.03 ( 0.37 to 11.09$), \mathrm{P}=0.40)$ and in venous thromboembolism (84.3 v 35.3 per 10000 womenyears, hazard ratio 2.39 (0.62 to 9.24$), \mathrm{P}=0.19$ ).

\section{Adverse events}

There were 15 deaths during the trial, with a non-significant increase in the rate in the combined therapy group compared with placebo (30.7 $v 19.2$ per 10000 womenyears, hazard ratio 1.60 (0.52 to 4.89$))$. Serious adverse events by diagnostic category and treatment group are

\begin{tabular}{|c|c|c|c|c|}
\hline \multirow[b]{2}{*}{ Serious adverse events } & \multicolumn{2}{|c|}{ Combined therapy $v$ placebo* } & \multicolumn{2}{|c|}{ Combined therapy $v$ oestrogen therapy $\dagger$} \\
\hline & $\begin{array}{l}\text { Combined therapy } \\
\qquad(n=2196)\end{array}$ & Placebo $(n=2189)$ & $\begin{array}{l}\text { Combined therapy } \\
\qquad(\mathrm{n}=815)\end{array}$ & $\begin{array}{l}\text { Oestrogen therapy } \\
\qquad(n=826)\end{array}$ \\
\hline Visual disturbance & 19 & 33 & 12 & 5 \\
\hline Gallbladder disease & 13 & 13 & 10 & 10 \\
\hline Genitourinary & 9 & 7 & 3 & 1 \\
\hline Cardiac and vascular & 9 & 7 & 1 & 3 \\
\hline Musculoskeletal and rheumatic & 7 & 18 & 7 & 3 \\
\hline Gastrointestinal & 5 & 10 & 4 & 3 \\
\hline Other ophthalmic and neuropsychiatric & 5 & 3 & 1 & 0 \\
\hline Other hepatobilliary disorders & 2 & 3 & 1 & 2 \\
\hline Respiratory and ear, nose, and throat & 2 & 6 & 0 & 6 \\
\hline Haematological and endocrine & 2 & 4 & 1 & 0 \\
\hline Dermatological & 1 & 3 & 0 & 1 \\
\hline Miscellaneous symptoms and other & 0 & 2 & 1 & 4 \\
\hline All adverse events & 74 & 109 & 41 & 38 \\
\hline
\end{tabular}

*Combined therapy = conjugated equine oestrogen $0.625 \mathrm{mg}+$ medroxyprogesterone acetate $2.5 \mathrm{mg}$ orally daily. tOestrogen therapy = conjugated equine oestrogen $0.625 \mathrm{mg}$ orally daily. 
listed in table 5. There was no excess of serious adverse events in either of the randomised comparisons.

\section{DISCUSSION}

Data from WISDOM suggest that women starting or restarting combined oestrogen and progestogen therapy an average of 14 years after menopause are at increased risk of cardiovascular disease and venous thromboembolism, at least in the early years of treatment. We found a trend towards a decreased risk of osteoporotic fracture and no difference in the risk of stroke or cancers. The small numbers of events and the brief follow-up periods inevitably mean that some of the results cannot be confidently interpreted. However, we can comment on the results for early cardiovascular and thromboembolic disease. In a direct comparison with combined oestrogen and progestogen therapy, oestrogen only therapy may have similar, but smaller, short term effects. These results are consistent with the findings of the combined oestrogen and progestogen therapy arm of the women's health initiative study and with secondary prevention trials, and support the conclusion that combined oestrogen and progestogen therapy should not be given for cardiovascular disease prevention in older postmenopausal women. ${ }^{4-9}$

\section{Value of study}

Despite the fact that WISDOM did not run to completion, this trial makes an important contribution to the body of knowledge about hormone replacement therapy started in older postmenopausal women of a mean age of 63 years. The WISDOM population was recruited from women on general practice registers in countries where health care is free or with low fees. The randomised women had a similar health profile, with regard to the factors of interest in the trial, as those in the same age range who were first screened. With the exception of a history of breast cancer, a family history of venous thromboembolism, and other exclusion factors that would have made them less likely to take hormone replacement therapy, the WISDOM participants were similar to the registered population and to the UK population of the same age range. ${ }^{19} \mathrm{~A}$ strength of the study is that participants are likely to be representative of the general population of women of this age and the results applicable to this older age group. Comparing the population in the women's health initiative study, many of the women in WISDOM were similarly overweight or obese and had many similar cardiovascular risk factors. $^{72}$ The mean age at entry was also similar. However, previous use of hormone replacement therapy was higher in WISDOM than in the women's health initiative study $(45 \%$ compared with $26 \%$ in women with a uterus).

\section{Comparison of results}

For combined oestrogen and progestogen therapy, the WISDOM results are similar to the findings in the early years of the women's health initiative trial.
Venous thromboembolism-The event rate in women taking combined oestrogen and progestogen therapy in WISDOM was higher than that reported in the early years of the women's health initiative trial, despite the exclusion in WISDOM of those with previous events. The reason for this is not clear but, in view of the small number of events, may be a chance finding. The increased rate of venous thromboembolism with combined therapy was greatest in the first year of the women's health initiative trial. ${ }^{7}$ It is possible that those with genetic predisposition to thrombosis have early vulnerability to hormone replacement therapy. ${ }^{20}$

Cardiovascular disease-Although the number of cardiovascular events observed was small, all occurred in the hormone replacement therapy groups, at a rate of 27 per 10000 women-years in the combined therapy arm of WISDOM. This rate was smaller than the rate of 51 per 10000 women-years in the first year of the combined therapy arm of the women's health initiative study. The early increased risk of cardiovascular events in both trials is compatible with the hypothesis that administration of hormone replacement therapy, particularly combined oestrogen and progestogen therapy, to women many years after menopause, who are likely to have established atherosclerosis, may cause disruption of the plaque surface, with subsequent platelet adhesion, clotting, and further arterial narrowing. ${ }^{21}$ Most of the events in WISDOM occurred in women over the age of 64 , many of whom had cardiovascular risk factors.

Fractures-The non-significant trend toward a reduced risk of fractures after an average follow-up period of only one year is in keeping with the significant reduction of fractures seen in the women's health initiative study. ${ }^{72}$ Neither WISDOM nor the women's health initiative study required an increased risk of fracture as an inclusion criterion, and so the results suggest a potent preventive effect in an unscreened population. As in the women's health initiative study, we found no apparent difference between combined therapy and oestrogen therapy in their effect on fracture prevention.

Cancer-We found no effect on cancer rates, including breast cancer, but this must be interpreted with caution as the maximum follow-up was three years (median one year). A decrease in the annual risk of breast cancer of 7 per 10000 cases compared with placebo after nearly seven years of oestrogen therapy was reported in the women's health initiative study, which approached statistical significance, but combined oestrogen and progestogen therapy was associated with a significantly increased annual risk of 8 per 10000 cases after five years. $^{7222}$ WISDOM was unable to shed light on the relation between progestogen and breast cancer, although the data for all outcomes suggested the greater safety of oestrogen only therapy, similar to the results from the women's health initiative trial.

Death-The apparent but non-significant increase in mortality in users of combined therapy versus placebo is not in keeping with a recent meta-analysis of all randomised controlled trials of hormone replacement 


\section{WHAT IS ALREADY KNOWN ON THIS TOPIC}

Combined oestrogen and progestogen hormone replacement therapy initiated many years after menopause in asymptomatic women reduces fracture risk but increases thromboembolic, breast cancer, and possibly cerebrovascular risk

Oestrogen only hormone replacement therapy started near the menopause may decrease the risk of coronary heart disease, breast cancer, diabetes, and osteoporotic fractures

\section{WHAT THIS STUDY ADDS}

This study confirms an early increase in thromboembolic and cardiovascular risk in women starting hormone replacement therapy at a mean of 63 years and 15 years after the menopause

These uncommon serious events must be weighed against more common improvements in quality of life

These results cannot be applied to symptomatic women starting hormone replacement therapy near menopause, for whom cardiovascular benefits have recently been described

therapy (including the women's health initiative), which showed no increased mortality overall and a significantly reduced mortality in hormone users younger than 60 years (odds ratio 0.67 (95\% confidence interval 0.49 to 0.92$)) .{ }^{23}$ The short follow-up time and small number of deaths recorded in WISDOM do not allow robust conclusions.

\section{Limitations of study}

The follow-up period in WISDOM was short because of the early closure, and the power of the study was greatly reduced by the curtailed recruitment, which also led to relatively few women being in the younger age groups. No conclusions can be drawn about the outcomes in relation to these age groups.

\section{Implications of results}

In WISDOM only two hormone replacement therapy regimens were studied, which contained conjugated equine oestrogens and, when combined, medroxyprogesterone acetate. The medical profession and the media must interpret and disseminate the results of WISDOM with caution and responsibility. In 2002 unconsidered and sometimes exaggerated responses to the first report from one arm of the women's health initiative study caused great alarm and distress to women around the world, with many suddenly stopping their hormone replacement therapy without medical consultation and in some cases with adverse consequences. ${ }^{2425}$

The results of WISDOM, like those of the women's health initiative study, help test the hypothesis that starting long term hormone replacement therapy in elderly, often asymptomatic, women in their $60 \mathrm{~s}$ might reduce major morbidities, in particular cardiovascular disease. With the exception of fractures, this does not seem likely. However, currently, such women rarely start taking hormone replacement therapy at these ages. Most women who start hormone replacement therapy do so near the menopause to reduce menopausal symptoms and improve their quality of life. Clinical and animal studies suggest that the effect of oestrogen on the cardiovascular system and possibly the brain may be very different and probably beneficial when used at or near the time of menopause. ${ }^{26-28}$ In particular, a recent meta-analysis of 23 randomised controlled trials of hormone replacement therapy showed that it significantly reduced coronary heart disease in women starting therapy younger than 60 years or within 10 years of menopause. ${ }^{29}$ The early termination of WISDOM before large numbers of recently menopausal women could be recruited, means that the "critical window hypothesis" could not be examined to see if oestrogen has cardioprotective and neuroprotective effects. ${ }^{2030}$ The risk: benefit equation for a younger menopausal woman may be different from that seen in the mainly older women in WISDOM and the women's health initiative study.

The publicity surrounding the first report from the first arm of the women's health initiative study put great pressure on the funders of WISDOM to stop this trial. ${ }^{1531}$ Details of the more favourable results from the oestrogen only arm and the reanalyses of the women's health initiative study by age and years from menopause, which suggested different results when hormone replacement therapy is started in early menopause, became available only several years after the closure of WISDOM. ${ }^{32}$ A long term, randomised, placebo controlled trial of hormone replacement therapy from menopause is still needed but presents great problems in terms of funding, compliance, and continuance, especially in symptomatic women.

\section{Conclusions}

The women's health initiative study and WISDOM have not answered the question about long term benefits and risks of hormone replacement therapy in the large majority of women who start therapy around menopause for symptom control. However, they have shown that there is no overall disease prevention benefit, and some potential risk, for women with few or no oestrogen deficiency symptoms who start hormone replacement therapy many years after menopause. If there is a menopausal window of therapeutic benefit its upper limit has not been well defined and is likely to vary with arterial health and associated risk factors such as obesity and metabolic syndrome. ${ }^{33}$

Both the women's health initiative study and WIS DOM were specifically looking for chronic disease prevention in older postmenopausal women who did not have disabling menopausal symptoms. An increased quality of life associated with a reduction in menopausal symptoms is the usual motivation for taking hormone replacement therapy. Quality of life data from WISDOM will be published separately, and these results should be considered alongside the main morbidity and mortality outcome data described in this paper. Those helping women make choices about treatment should consider both the results and limitations of the women's health initiative study and WISDOM, particularly those that may be influenced by the timing of starting hormone replacement therapy. 
We thank all the research nurses, general practitioners, practice staff, and, in particular, the women who kindly participated in WISDOM.

Contributors: MRV was the principal investigator for WISDOM; wrote the application for funding with HCW and TWM; grantee of UK funding; responsible for trial design, protocol development, trial procedures, and overall management; contributed to data analysis and interpretation; and drafted the paper and contributed to the final report. AHM was the principal investigator in Australia; contributed to trial design, trial procedures, analyses, and interpretation of data; grantee of Australian funding; and corresponding author of final paper. BL was the co-principal investigator in New Zealand; contributed to the trial design, trial procedures, interpretation of data; and management of trial conduct and cessation; grantee of New Zealand funding. DF contributed to the trial protocol, trial procedures, data management, analyses and interpretation of data, and writing of the final report. JM was responsible for training and coordination of UK research nurses and quality control and contributed to the analysis and writing of the final report. SKM was coinvestigator and clinical epidemiologist; contributed to trial design, trial procedures, analyses and interpretation of data, and writing the final report BLDeS supervised data management and carried out statistical analyses and contributed to presentation and interpretation of the results and to writing of the final report. SR was co-investigator and project manager of the trial in New Zealand; contributed to the trial design, interpretation of data, and management of trial conduct and cessation. AD was joint principal investigator in New Zealand; contributed to trial design, trial procedures, interpretation of data, and management of trial conduct and cessation. HCW was responsible for statistical design including pilot studies, trial protocol and procedures, data management analysis and writing of report; UK funding grantee. JHD was principal investigator in UK after retirement of TWM; contributed to later trial management, data analysis, and writing of final report. TWM was principal investigator in UK and grantee of UK funding; contributed to trial design, management, and writing of the final report.

Other members of the WISDOM team. Abdalla M, statistical analysis, Department of Epidemiology and Population Health, London School of Hygiene and Tropical Medicine. Allen P, Balen H, Bastick R, Brown H, Foulger K, Fox S, Glynn V, Hall A, Hand L, Hill A, Leathem C, Mackinnon W, Marshall E, Williams A, regional training nurses; Collins N, O'Conner B, trial managers; Ghali M, Furness P, Islam MZ, software design for electronic data collection and management; Harrild K, statistical analysis and data management; Knott C, Taylor L, implementation of trial protocol and management, research nurse support; Walgrove MA, contributed to protocol, developed manual, nurse support; Welton Al, contributed to trial protocol and management; Zhu C-Q, statistician; Zuhrie SR, development of trial protocol and medical advice; MRC General Practice Research Framework, London. Griffith E, data manager and pharmacy control; Ryan P, co-investigator in South Australia, co-grantee of Australian funding; Discipline of Public Health, University of Adelaide, Australia. Komesaroff P, senior investigator in Victoria, University of Monash, Victoria, Australia. Marley J, senior investigator in NSW and co-grantee of Australian funding, University of Newcastle, New South Wales, Australia. Paine BJ, trial coordinator, Discipline of Obstetrics and Gynaecology, University of Adelaide. Stocks NP, medical director, Discipline of General Practice, University of Adelaide.

Sources of funding: UK Medical Research Council, British Heart Foundation, Department of Health for England, Scottish Office, Welsh Office, Department of Health and Social Services for Northern Ireland, Royal Australian and New Zealand College of Obstetricians and Gynaecologists, Australasian Menopause Society, National Health and Medical Research Council (NHMRC), National Heart Foundation of Australia, The Cancer Council of South Australia, The Cancer Society of New Zealand (Wellington Branch), NHS R\&D Executive (service support and excess treatment costs). MRC, in collaboration with the other UK funders, established a Trial Steering Committee (which reported annually to the MRC) with an independent chairman and independent Data Monitoring and Ethics Committee (which considered unblinded group data, took account of external developments relevant to the progress of the trial, and made recommendations to the steering committee). The funders in Australia and New Zealand monitored local progress and received reports on progress from the UK steering committee. The principal investigators from Australia and New Zealand were non-voting members of the UK steering committee. Competing interests: WISDOM was run and funded independently of industry. The funding bodies had no influence on the results except for the early curtailment of the trial by the MRC. Wyeth Ayerst provided active drugs and matched placebo but had no other involvement in the trial. All authors have declared no direct conflicts of interest. AHM and BL have received research grants and lecture honoraria from a variety of industry sources not associated with WISDOM.

Ethical approval: UK approval was granted by the South Thames Regional Health Authority Multicentre Research Ethics Committees and by the relevant local research ethics committees. Australian approval was given by the human research ethics committees for the universities of Adelaide, Newcastle, and Monash and by the Royal Australian College of General Practitioners' National Research and Evaluation Ethics Committee. New Zealand approval was given by the Wellington Regional Ethics Committee and the Auckland and Canterbury ethics committees.

1 MacLennan AH, Broadbent IL, Lester S, Moore V. Oral oestrogen and combined oestrogen/progestogen therapy versus placebo for hot flushes. Cochrane Database Syst Rev 2004;(4):CD002978.

2 Suckling J, Lethaby A, Kennedy R. Local oestrogen for vaginal atrophy in postmenopausal women. Cochrane Database Syst Rev 2003;(4): CD001500.

3 Moehrer B, Hextall A, Jackson S. Oestrogens for urinary incontinence in women. Cochrane Database Syst Rev 2003;(2):CD001405.

4 Hulley S, Grady D, Bush T, Furberg C, Herrington D, Riggs B, et al. Randomized trial of estrogen plus progestin for secondary prevention of coronary heart disease in postmenopausal women. Heart and Estrogen/progestin Replacement Study (HERS) Research Group. JAMA 1998;280:605-13.

5 Hoibraaten E, Qvigstad E, Arnesen H, Larsen S, Wickstrom E, Sandset PM. Increased risk of recurrent venous thromboembolism during hormone replacement therapy. Results of the randomized, double-blind, placebo-controlled estrogen in venous thromboembolism trial (EVTET). Thromb Haemost 2000;84:961-7.

6 Viscoli CM, Brass LM, Kernan WN, Sarrel PM, Suissa S, Horwitz RL. A clinical trial of estrogen-replacement therapy after ischemic stroke. N Engl J Med 2001:345:1243-9.

7 Writing Group for the Women's Health Initiative Investigators. Risks and benefits of estrogen plus progestin in healthy postmenopausa women. Principal results from the women's health initiative randomized controlled trial. JAMA 2002;288:321-33.

8 Clarke SC, Kelleher J, Lloyd-Jones H, Slack M, Schofield PM. A study of hormone replacement therapy in postmenopausal women with ischaemic heart disease: the Papworth HRT atherosclerosis study. BJOG 2002;109:1056-62.

9 ESPRIT Team. Oestrogen therapy for prevention of re-infarction in postmenopausal women: a randomised controlled trial. Lancet 2002;359:2001-8.

10 Waters DD, Alderman EL, Hsia J, Howard BV, Cobb FR, Rogers WJ, et al. Effects of hormone replacement therapy and antioxidant vitamin in postmenopausal supplements on coronary atherosclerosis in postmenopausal women: a randomized controlled trial. JAMA 2002;288:2432-40.

11 Holmberg L, Anderson H, for the HABITS steering and data monitoring committees. HABITS (hormonal replacement therapy after breast cancer-is it safe?), a randomised comparison: trial stopped. Lancet 2004:363:453-5.

12 Women's Health Initiative Steering Committee. Effects of conjugated equine estrogen in postmenopausal women with hysterectomy. The women's health initiative randomised controlled trial. JAMA 2004:291:1701-12.

13 Veerus P, Hovi S, Fischer K, Rahu M, Hakama M, Hemminki E. Results from the Estonian postmenopausal hormone therapy trial. Maturitas 2006;55:162-73.

14 Manson JE, Hsia J, Johnson KC, Rossouw JE, Assaf AR, Lasser NL, et al. Estrogen plus progestin and the risk of coronary heart disease. $N$ Engl J Med 2003:349:523-34.

15 Vickers M. The MRC long-term randomised controlled trial of hormone replacement therapy: background, design and objectives. I Br Menopause Soc 1996;1:9-13.

16 Vickers M, Meade T, Darbyshire J. WISDOM: history and early demise - was it inevitable? Climacteric 2002;5:317-25.

17 Vickers MR, Martin J, Meade TW and the WISDOM Study Team. The women's international study of long duration oestrogen after menopause (WISDOM): a randomised controlled trial. BMC Women's Health 2007;7:2. www.biomedcentral.com/1472-6874/7/2.

18 Collett D. Modelling survival data in medical research. London: Chapman and Hall, 1994.

19 Bajekal M. Use of health services and prescribed medicines. In Department of Health. Health survey for England 1998. www.archive. official-documents.co.uk/document/doh/survey98/hse-07.htm.

20 Cushman M, Kuller LH, Prentice R, Rodabough RJ, Psaty BM, Stafford RS, et al. Oestrogen plus progestin and risk of venous thrombosis. JAMA 2004;292:1573-80.

21 Phillips LS, Langer RD. Postmenopausal hormone therapy: critical reappraisal and a unified hypothesis. Fertil Steril 2005:83:558-66.

22 Stefanick ML, Anderson GL, Margolis KL, Hendrix SL, Rodabough RJ, Paskett ED, et al. Effects of conjugated equine estrogens on breast 
cancer and mammography screening in postmenopausal women with hysterectomy. JAMA 2006;295:1647-57.

23 Salpeter S. Mortality associated with hormone replacement therapy in younger and older women. J Gen Intern Med 2004;19:791-804.

24 Lawton B, Rose S, McLeod D, Dowell AC. Changes in use of hormone replacement therapy after the report from the women's health initiative: cross sectional survey of users. BMJ 2003;327:845-6.

25 MacLennan AH, Taylor AW, Wilson DH. Hormone therapy use after the women's health initiative. Climacteric 2004;7:138-42.

26 MacLennan AH, Henderson VW, Paine BJ, Mathias J, Ramsay EN, Ryan P, et al. Hormone therapy, timing of initiation, and cognition in women older than 60 years: the REMEMBER pilot study. Menopause 2006;13:28-36.

27 Clarkson TB, Appt SE. Controversies about HRT-lessons from monkey models. Maturitas 2005;51:64-74.

28 Grodstein F, Manson JE, Stampher MJ. Hormone therapy and coronary heart disease: the role of time since menopause and age at hormone initiation. J Women's Health 2006;15:35-44.
29 Salpeter SR, Walsh JME, Greyber E, Salpeter EE. Coronary heart disease events associated with hormone therapy in younger and older women. J Gen Intern Med 2006;21:363-6.

30 Salpeter S. Hormone therapy for younger postmenopausal women: how can we make sense out of the evidence? Climacteric 2005;8:307-10.

31 MacLennan AH, Sturdee D. The end of WISDOM. Climacteric 2002;5:313-6.

32 Manson JE, Allison MA, Rossouw JE, Carr JJ, Langer RD, Hsia J, et al. Estrogen therapy and coronary-artery calcification. N Engl J Med 2007;356:2591-602.

33 Tankó LB, Christiansen C. Adipose tissue, insulin resistance, and low-grade inflammation: implications for atherogenesis and the cardiovascular harm of estrogen plus progestogen therapy. Climacteric 2006;9:169-80.

Accepted: 2 July 2007 